\title{
HACIA LA COMPRENSION DE LA ESCUELA Y SUS VIVENCIAS * Proyectos de Renovación Pedagógica para la Escuela Primaria
}

\author{
Gladys Amaya R., María del Pilar Unda B., \\ Germán Vargas G. - Director de Tesis -
}

El presente estudio tiene la intención de brindarle un espacio al maestro para que se pronuncie sobre temas que conoce y, sobre todo, que sabe por su experiencia vivencial. A su vez, ha nacido de la convicción de que ya es tiempo de que el investigador vea en el saber docente (o del docente) una fuente viva, válida y llena de matices para profundizar en el conocimiento de la cotidianeidad de la escuela.

Es preciso, de antemano, advertir una insuficiencia: la toma de la palabra por el maestro no puede venir de que alguien (llámese investigador, directivo, capacitador) le diga que hable; la verdadera voz del maestro será dicha cuando él se pronuncie, cuando se entienda como el hacedor de la educación y protagonista del conocimiento pedagógico. Aquí el maestro y los demás miembros de la "comunidad educativa" fueron invitados al pronunciamiento sobre la escuela.

No tenemos su voz auténtica, pero nos permite tener -en lo que han dicho- un punto de referencia para animar procesos donde tomen ellos efectivamente su papel protagónico.

Este estudio se incrusta dentro de un ideal metodológico que apenas está vislumbrado por la investigación educacional (y en general por la investigación social): se busca la recuperación crítica del conocimiento popular (en este caso docente) y la devolución sistemática. Así se intenta poner la investigación al servicio de los procesos que están, de hecho, siendo adelantados en un lugar y por unas personas concretas. No se quiso ver cómo se comportaba la realidad frente a la manipulación de algunas variables seleccionadas por los investigadores, sino que se vió un proceso educativo en curso y se le asistió para poder ofrecer en este momento una sistematización desde la mirada de investigadores sólo parcialmente comprometidos en el proceso. Esta manera de entender la investigación tiene que ser vista, todavía, como una esperanza o como una vía alterna y en parte con este estudio se quiere ofrecer un punto de referencia para pensar en ella.

Pero veamos más en su detalle la forma que se optó en este estudio: dado que los maestros del Distrito Especial de Bogotá están participando en un proceso de Renovación Pedagógica, se quiso ver algunos de los aspectos de ese proceso. Por eso se ha querido enfatizar al narrar la experiencia, en la serie de pasos que se dieron con esta mira desde la Dirección de Investigaciones Educativas, DIE-CEP. En segundo lugar, se intenta ver cómo piensan algunos de los participantes en el fenómeno educativo y confrontar lo que ellos plantean con algunos de los hallazgos que sobre los mismos temas ha cristalizado la investigación educacional en América Latina.

\footnotetext{
* El proyecto se desarrollé como una acción Institucional en la Dirección de Investigaciones Educativas (DIE) de la Secretaría de Educación del Distrito Especial de Bogotá. Conté con la dirección académica del Proyecto de Extensión REDUC-Colombia del Centro de Investigación de la Universidad Pedagógica Nacional (CIUP).

Sus resultados se han utilizado para la planeación institucional de la DIE.

Las autoras lo presentaron como Tesis para optar el título de Magister en Investigación Socio- Educativa y Curricular en la Universidad Pedagógica Nacional.
} 
Finalmente, se ha tratado de hacer una reflexión comprensiva del aporte que puede hacerse desde la teoría sobre el fenómeno observado. Con ello se quiso terminar y no comenzar el estudio pues, no se trata de describir "objetivamente" desde el investigador, sino de aportar una reflexión comprometida con el proceso que han adelantado los participantes en la Renovación Pedagógica. A esta reflexión es a lo que aludimos como devolución sistemática.

En este orden de lo conceptual el presente trabajo propone una discusión sistemática sobre el sentido social de la Educación, la escuela como una posibilidad de expresión de sentido (espacio donde se conoce y circula el conocimiento, se aclara y se contribuye a la decantación de valores para que los sujetos orienten su vida y si situen como sujetos históricos), el papel del maestro, la formación y capacitación como procesos de reproductivismo cultural, y, finalmente, la pedagogía como porvenir educativo en cuanto es capaz de transmitir y construir maneras de entender y explicar al hombre y la sociedad.

\section{PROPOSITOS DEL ESTUDIO}

1. Recuperar crítica y devolver sistemáticamente el saber del maestro sobre algunos aspectos de la Vida de la Escuela, analizando el modo como los sujetos la conciben y "explican".

2. Dinamizar la construcción de proyectos pedagógicos, desde una propuesta participativa que concibe al maestro como protagonista y constructor (más que como destinatario) de una propuesta de acción para su quehacer docente.

3. Promover la participación y compromiso del directivo Docente, buscando la recuperación de su rol pedagógico.

4. Contribuir al desarrollo de formas de articulación de lo investigativo con los procesos sociales, en los cuales se halla incerto el investigador.

5. Promover la construcción de comunidad educativa en torno a la escuela primaria, tomando como centro de interés del niño.

\section{ANTECEDENTES}

Un grupo de Supervisores de Primaria y Técnicos de la DIE-CEP, a lo largo de 1984, identificó algunos problemas en el abordaje de la escuela, fueron ellos: para la implementación de la Renovación Curricular se estaba priorizando la información sobre los programas, desplazando el intento de comprensión del niño; había poca incidencia de los programas de capacitación sobre la práctica de los maestro; se observó falta de sentido y coherencia en algunos programas de capacitación, se reconoció una desarticulación del proceso de planeación escolar, la parte burocrática del directivo docente primaba sobre el carácter pedagógico, y, era notorio el deficiente prestigio de la Educación Pública con su consecuente disminución en la matrícula y el registro de altas tasas de deserción y repitencia en la escuela.

A partir de la identificación de estos problemas, los técnicos de la DIE asumieron la función de animadores de la experiencia, en donde los primeros interlocutores habían de ser los supervisores de Primaria, de quienes se esperaba el ejercicio de una función multiplicadora entre los directores de escuela y maestros, cuya cobertura total es de 10.000 docentes. 


\section{CARACTERISTICAS DEL PROCESO DE REFLEXION - ACCION}

Cualquiera hubiera podido ser el punto de partida, el problema que compromete la reflexión, el desvelamiento progresivo de valores e intereses para iluminar y evaluar las acciones de manera que cada vez adquieran una mayor dimensión de sentido para quienes las realizan

En este caso, tomando como punto de referencia los problemas sobre los cuales ya los directivos docentes habían avanzado reflexiones y propuestas, y que se había evidenciado resultaba de gran interés en las escuelas -no en vano hicieron llegar más de mil propuestas de acción a partir del primer seminario con supervisores- se centró la atención sobre los problemas de deserción y repitencia que, como todos sabemos, afecta anualmente a miles de niños. Esto, por su parte, tenía una gran importancia para los directivos docentes con quienes se había detectado la necesidad de contrastar sus puntos de vista, los que habían expresado en la construcción de los proyectos, con los que pudiera preocupar e interesar a los maestros en sus escuelas. Es así como esta preocupación, que pudiera orientar solamente hacia la realización de tareas para la reducción de las cifras (a partir de la investigación y de la "evidencia" se han surgido actividades tales como aumento o disminución del tamaño de los grupos, heterogeneidad de los mismos, construcción de bibliotecas, disponibilidad de textos, etc.) generó un proceso de lectura subjetiva de la cotianeidad de la escuela preguntando en una primera instancia cómo se explican estos fenómenos, quiénes se encuentran ligados más directamente a los hechos, qué acciones se proponen desde la escuela para enfrentarlos. Desde allí, se espera avanzar en un proceso que permita clarificar los implícitos de las explicaciones y de las propuestas, y participar en la elaboración conjunta de Proyectos de Renovación Pedagógica, se obtuvo entonces información proveniente de 294 escuelas, lo que equivale a un $31.6 \%$ de la población total si se tiene en cuenta que actualmente existen 930. Sin embargo, a partir de ella sólo se puede decir que son las conclusiones de los maestros que han participado en dicho proceso; no es posible ni se pretende con ella afirmar que "así piensan los maestros", la población total del Distrito Especial de Bogotá.

Por otra parte, el propósito no es acumular "evidencia" o datos objetivos que permita a los miembros de la "comunidad científica" explicar el fenómeno de la deserción y de la repitencia, del bienestar o del malestar del niño en la escuela, de lo que facilita o dificulta su rendimiento. Lo que interesa aquí es recuperar la lectura subjetiva del ambiente escolar por quienes en ella participan porque, si bien desde un punto de vista "objetivo" determinados factores pueden estar explicando los fenómenos, éstos no adquieren ningún peso en la definción y dínamización de las acciones: lo que resulta importante en este sentido es aquello que es percibido como significativo por parte de quienes tienen y asumen la responsabilidad social de las mismas.

Para la sistematización se procedió de manera inductiva construyendo las categorías a partir de las respuestas de los maestros a las preguntas abiertas que se presentaron en el formato para la reflexión.

En los casos en que los profesores de diferentes niveles de una misma escuela sc refierieron a un mismo factor, en la tabulación se indicó simplemente la escuela con respecto al item correspondiente. Es decir, sería posible hacer un análisis por grados entre escuelas si se considera importante identificar problemáticas diferenciales por niveles; en esta sistematización interesaría una Visión más global de los fenómenos y por eso no se hizo. 
En el estudio se presentan cuadros que describen los tipos de atribución, la frecuencia y el porcentaje de escuelas que se refirieron a un determinado factor.

El porcentaje se obtuvo comparando el número de escuelas que identificó un determinado tipo de atribución con el número total de escuelas que responden ( $\mathrm{N}-294)$ para cada factor.

Para el análisis la información ha sido agrupada según la frecuencia de señalamiento de cada factor: hemos denominado Patrones de Atribución (P.A.) a los factores identificados como razón en más del $60 \%$ de las escuelas; y como juicios compartidos de atribución (J.C.A.) cuando entre el $20 \% 60 \%$ de la población lo señala.

\section{RESULTADOS}

En el trabajo se presenta un análisis crítico de las "explicaciones" de los maestros. Para explicar la deserción de los niños, los maestros se refieren fundamentalmente al cambio de domicilio, constituyendo éste el único P.A. y dejando sólo J.A.C distribuidos así: Para el área de matemáticas la falta de colaboración de los padres de familia, para Ciencias Naturales la falta de colaboración e interés del niño y en Ciencias Sociales la falta de motivación e interés del niño y la falta de material didáctico. A nivel general, la falta de motivación e interés de los niños y la falta de colaboración de los padres resultan ser entonces las principales "razones explicativas" de la repitencia.

Con respecto a los factores que dificultan el rendimiento del niño en la escuela, la falta de colaboración, desinterés e irresponsabilidad de los padres resulta ser un P.A. muy importante. Esto contrasta con las "explicaciones" para lo cual no se presentan P.A. sino J.C.A. referidos hacia el maestro mismo: su cumplimiento, interés y responsabilidad, la buena relación con los alumnos y la metodología empleada.

El bienestar del niño en la escuela es "explicado" por las relaciones que el maestro establece con sus alumnos, las actividades recreativas, deportivas y culturales que se realizan en la escuela y a los cuales se les asigna P.A., mientras el descontento es "explicado" fundamentalmente por las deficiencias de la planta física

A lo largo del trabajo, se hace un análisis crítico de esta información a la luz de la elaboración conceptual, contrastándola con los resultados de otras investigaciones que han pretendido una explicación causal de los fenómenos mencionados.

El aspecto más discutido es el que tiene relación con los resultados del análisis global, que muestra como los maestros atribuyen la deserción, repitencia y malestar del niño en la escuela a factores educativos, los cuales están referidos a aspectos macroestructurales, que giran alrededor de problemática social, familiar e institucional, mientras resultan ser los factores pedagógicos, los que en su opinión "explican" el bienestar y rendimiento del niño.

Finalmente, es imperante resaltar aquí que con este trabajo se pretende entregar una devolución crítica global de la información de un momento del proceso, que debe continuar, escuchando nuevamente la opinión del maestro sobre lo que aquí decimos. Se pretende así participar y contribuir para el avance de propuestas pedagógicas desde una mayor comprensión de la escuela y sus vivencias. 